\title{
CORRELAÇÕES ENTRE OS CARACTERES FÍSICO-QUÍMICOS DE FRUTOS DA ACEROLEIRA COM VARIÁVEIS METEOROLÓGICAS ${ }^{1}$
}

\author{
JOSÉ EVERALDO GOMES², DILERMANDO PERECIN³ E ANTÔNIO BALDO GERALDO MARTINS ${ }^{4}$
}

\begin{abstract}
RESUMO - Com objetivo de estimar as associações entre os caracteres físico-químicos do fruto com variáveis meteorológicas, conduziu-se um experimento no pomar da Faculdade de Ciências Agrárias e Veterinárias de Jaboticabal, no período de dezembro/97 a janeiro/99, avaliando-se em cada colheita espontânea de 5 genótipos, valores médios de altura e diâmetro de vinte frutos, teor de vitamina $\mathrm{C},{ }^{\circ} \mathrm{BRIX}, \mathrm{pH}$, rendimento médio de polpa em três amostras de vinte frutos, massa média de frutos e tempo de colheita em dias. Os dados meteorológicos diários utilizados foram obtidos na Estação Agroclimatológica do Departamento de Ciências Exatas da FCAV/ UNESP. Baseando-se nas maiores correlações entre as médias das temperaturas máxima (TMAX), mínima (TMIN) e média (TME) no período de colheita (1), no período de colheita incluindo-se os três dias antecedentes (2) e no período de colheita incluindose os dez dias antecedentes (3), associadas com os caracteres físico-químicos de frutos, elegeu-se TMIN2, como a mais influente entre as estudadas e estimou-se o somatório da precipitação (PR) e horas-luz diárias (HL) do mesmo período. Com as estimativas de precipitação e insolação em horas-luz diárias e TMIN2, obtiveram-se as correlações entre os caracteres físico-químicos de frutos de aceroleiras na média geral e dentro da média de cada genótipo analisado. Concluiu-se que as variáveis meteorológicas TMIN2, PR e HL apresentaram respostas diferenciadas dentro de cada genótipo, influenciando as expressões dos caracteres físico-químicos de seus frutos e que as correlações entre HL e tempo de colheita em dias, entre e dentro de todos os genótipos estudados, foram direta e altamente significativas, de modo que a variável HL condicionou a periodicidade e extensão das colheitas (safras).
\end{abstract}

Termos para indexação: métrico-tecnológico, Malpighia emarginata DC., insolação.

\section{CORRELATIONS AMONG PHYSICO-CHEMICAL CHARACTERS OF WEST INDIAN CHERRY FRUITS WITH METEOROLOGICAL VARIABLES}

\begin{abstract}
The objective of this experiment was to estimate associations among physico-chemical characters with meteorological variables from December/97 to January/99 in FCAV, Jaboticabal, SP. At every spontaneous crop of 5 genotypes, medium height and diameter of twenty fruits, vitamin $\mathrm{C}$ amount, Brix, $\mathrm{pH}$, medium pulp profit in three samples of twenty fruits, medium meight of fruits and crop time were evaluated. Daily meteorological data were taken from the agrometeorological Department of Exact Sciences in FCAVUNESP. Supported by the largest correlations among averages of maximum (TMAX), minimum (TMIN) and medium (TME) temperatures at the crop period (1), at the crop period plus three days before it (2) and at the crop period plus ten days before it (3) associated to the physico-chemical characters of fruits, TMIN2 was chosen as the most effective in correlated responses, and data from precipitacion (PR) and daily sunny hours (HL) in the same period (TMIN2) were comprised. With precipitacion and daily sunny hours data from TMIN2 period, correlations with physico-chemical characters of West Indian cherry fruits were measured in general average and in the average inside each genotype. It could be concluded that meteorological variables TMIN2, PR, HL showed different responses inside genotypes and influenced physico-chemical characters of fruits; HL correlation to crop time among and inside the genotypes were directly and highly significant and the variable HL influenced the periodicity and extention of crops.
\end{abstract}

Index terms: metric-technological, Malpighia emarginata DC, insolation.

\section{INTRODUÇÃO}

A cultura da acerola (Malpighia emarginata D.C.) tornou-se economicamente importante e adquiriu status de pomar comercial desde a descoberta do alto conteúdo de vitamina $\mathrm{C}$ de seus frutos. Semelhantemente a outras frutíferas, a produtividade e a qualidade dos frutos da aceroleira podem ser influenciadas pela seca, frio, vento, calor, pragas, doenças e estado nutricional das plantas.
O estudo das correlações entre os caracteres físicoquímicos do fruto da aceroleira com as variáveis meteorológicas é de importância, pois pode fornecer subsídios para a definição de um sistema de produção compatível com as diversas regiões agroclimáticas.

A produção de ácido ascórbico é bastante influenciada pela radiação solar (Murphy, 1939; Harding et al.,1939; Winstron, 1947; e Brown, 1955). Asenjo (1959) aponta a elevada altitude como provável causa do baixo conteúdo de ácido ascórbico nas

1 (Trabalho 065/2000). Recebido: 16/05/2000. Aceito para publicação: 08/02/2002. Extraído da tese de Doutoramento em desenvolvimento na FCAV/ UNESP. Financiada pela Fundação de Amparo à Pesquisa do Estado de São Paulo (FAPESP).

2 Biólogo M. Sc., Bolsista FAPESP, Dep. Ciências Exatas da FCAV/ UNESP, Rod. Prof. Paulo Donato Castelane s/n, Cep:14.870-000, Jaboticabal, SP.

3 Eng. Agr., Prof. Titular, Dep. de Ciências Exatas da FCAV/UNESP, Rod. Prof. Paulo Donato Castelane s/n, Cep:14.870-000, Jaboticabal, SP. Email:perecin@.unesp.br. Bolsista CNPq.

4 Eng. Agr. Prof. Assistente Dr. Dep. de Produção Vegetal da FCAV/UNESP, Rod. Prof. Paulo Donato Castelane s/n, Cep:14.870-000, Jaboticabal, SP.Email:baldo@.fcav.unesp.br. 
aceroleiras da Guatemala, uma vez que frutas com altos teores de ácido ascórbico encontram-se em regiões cuja altitude não ultrapassa a $1000 \mathrm{~m}$. Nakasone et al. (1966), no Havaí, analisaram o efeito da radiação solar no conteúdo de ácido ascórbico em frutos de aceroleiras e, trabalhando em cinco níveis de luminosidade, constataram redução de $17 \%$ no teor de ácido ascórbico em plantas com $75 \%$ de sombreamento, quando comparadas àquelas que não sofreram restrição da luz solar no mesmo período de observação. Aróstegui et al. (1955) observaram que ocorre relação entre os fatores climáticos e o teor de vitamina $\mathrm{C}$ dos frutos da aceroleira e que diferenças do teor dessa vitamina nos frutos colhidos em épocas distintas são consideráveis, merecendo as causas, investigações detalhadas. Estudos realizados por Jackson \& Pennock (1958), em Porto Rico, em dez clones selecionados de aceroleiras, indicaram que safras mais longas e produtivas, totalizando $96 \%$ da produção anual, ocorreram de maio a outubro, meses mais quentes e chuvosos. Teixeira et al. (1995) classificaram como ótima para o desenvolvimento e produtividade comercial da aceroleira a faixa de temperatura entre 25 e $27^{\circ} \mathrm{C}$, podendo a planta ser cultivada em regiões com temperatura média igual ou superior a $20^{\circ} \mathrm{C}$ e temperatura média do mês mais frio maior ou igual a $14^{\circ} \mathrm{C}$; estabeleceram, ainda, o ideal de $2000 \mathrm{~mm}$ como limite máximo e $1200 \mathrm{~mm}$ como limite mínimo de precipitação média anual, considerando a região de dispersão natural. González (1996), estudando a biologia floral de dois acessos de Passiflora cincinnata Mast. em Jaboticabal-SP, concluiu que a redução das temperaturas e do fotoperíodo prejudicou o desenvolvimento do botão floral e o número de flores que atingiram a abertura.

Objetivou-se estimar as associações existentes entre os caracteres físico-químicos do fruto da aceroleira com as variáveis meteorológicas, com base na média geral dos genótipos e dentro de cada um estudado.

\section{MATERIAL E MÉTODOS}

O experimento, para estimar as associações existentes entre os caracteres físico-químicos do fruto da aceroleira com as variáveis meteorológicas foi conduzido no pomar da Faculdade de Ciências Agrárias e Veterinárias de Jaboticabal-SP (FCAV), situada a $21^{\circ} 5^{\prime} 22^{\prime \prime}$ de latitude sul, $48^{\circ} 18^{\prime} 58^{\prime \prime}$ de longitude oeste e altitude de $575 \mathrm{~m}$. Selecionaram-se para as avaliações cinco genótipos (CL2, CL5, CL11, R1 e R2), segundo análises prévias dos caracteres de rendimento, massa e teor de vitamina $\mathrm{C}$ dos frutos.

Os dados meteorológicos diários utilizados no trabalho foram obtidos na Estação Agroclimatológica do Departamento de Ciências Exatas da FCAV, UNESP.

Os estudos foram desenvolvidos de dezembro/97 a janeiro/99, avaliando-se, em cada colheita espontânea dos genótipos de aceroleiras, os seguintes caracteres de seus frutos: altura média de vinte frutos (TMFR), diâmetro médio de vinte frutos (DMFR), ambos em centímetros, teor de vitamina C (VITC) pelo método de redução do iodo $\mathrm{em} \mathrm{mg} / 100 \mathrm{ml}$ de suco (Instituto Adolfo Lutz, 1985), sólidos solúveis totais ( $\left.{ }^{\circ} \mathrm{BRIX}\right)$, pela medida refratométrica de Pearson (1973), $\mathrm{pH}$, através de um peagâmetro (Instituto Adolfo Lutz, 1985), rendimento médio de polpa em três amostras de vinte frutos, em porcentual (RMP20FR), massa média de frutos numa amostra de vinte frutos, em gramas (PMFR) e tempo de colheita em dias, calculado desde a coleta do primeiro até a colheita do último fruto (TCOLH).

A partir das observações diárias dos dados meteorológicos, considerando-se as temperaturas máxima (TMAX), mínima (TMIN) e média (TME), foram estimadas a média de temperatura máxima no período de colheita (TMAX1), a média de temperatura máxima, incluindo-se o período de colheita e os três dias antecedentes (TMAX2), e a média de temperatura máxima, incluindo-se o período de colheita e os dez dias antecedentes (TMAX3); a mesma metodologia foi adotada na estimativa da média de temperatura mínima, no período de colheita (TMIN1), a média de temperatura mínima, incluindo-se o período de colheita e os três dias antecedentes (TMIN2), a média de temperatura mínima, incluindo-se o período de colheita e os dez dias antecedentes (TMIN3), a média de temperatura média, no período de colheita (TME1), a média de temperatura média, incluindo-se o período de colheita e os três dias antecedentes (TME2), e a média de temperatura média, incluindo-se o período de colheita e os dez dias antecedentes (TME3). A partir dos valores médios de temperatura encontrados, foram obtidas, com auxílio do PROC CORR do SAS (SAS/STAT, 1995), as correlações entre essas variáveis e os caracteres físico-químicos do fruto dos cinco genótipos de aceroleiras, tanto para a média geral dos genótipos como para cada genótipo estudado.

\section{RESULTADOS E DISCUSSÃO}

A média de temperatura mínima obtida no período da colheita, juntamente com os três dias antecedentes (TMIN2), foi eleita dentre as demais como a mais influente, entre os períodos estudados, por apresentar os maiores valores de correlações com as variáveis físico-químicas dos frutos. A partir daí, tomaramse os dados totais de precipitação (PR) e insolação em horas-luz diárias (HL), do mesmo período, para obter as correlações entre as variáveis meteorológicas e os caracteres físico-químicos de frutos de aceroleiras que, apresentadas nas Tabelas $1 \mathrm{e} 2$, refletem os valores entre todos os genótipos e dentro de cada genótipo, respectivamente.

Observou-se que a média de temperatura mínima obtida, incluindo-se o período de colheita e os três dias antecedentes (TMIN2), associou-se positivamente (embora com valores baixos e próximos do limite de significância) com altura média de frutos (TMFR), diâmetro médio de frutos (DMFR), rendimento médio de polpa em três amostras de vinte frutos (RMP20FR) e massa média de frutos (PMFR), de modo que a redução (ou aumento) da TMIN2 tende a resultar na redução (ou aumento) destes caracteres, esclarecendo em parte as variações de tamanho de frutos que ocorrem ao longo das colheitas. Observa-se, na Tabela 1, o valor de 0,38 para correlação de TMIN2 com o rendimento médio de polpa em três amostras de vinte frutos (RMP20FR); embora significativo, é um valor baixo, sugerindo que outros fatores afetam o rendimento. Verifica-se também a sensibilidade do ${ }^{\circ}$ BRIX às variações de temperatura, pois os coeficientes de correlações de $-0,52$ (Tabela 1) e de $-0,77$ no genótipo CL5 (Tabela 2) são ambos significativos a $1 \%$ e $5 \%$ de probabilidade, respectivamente.

O somatório de precipitação (PR) obtido no período de 
TABELA 1 - Correlações entre a média geral dos caracteres físico-químicos de frutos de aceroleiras analisadas (CL2, CL5, CL11, R1 e R2) e as variáveis meteorológicas no período de dezembro/97 a janeiro/99, nas condições de Jaboticabal-SP.

\begin{tabular}{c|c|c|c|c|c|c|c|c|}
\hline \multirow{2}{*}{$\begin{array}{c}\text { Variáveis } \\
\text { Meteorológicas }\end{array}$} & \multicolumn{7}{|c|}{ Caracteres fisico-químicos em frutos de aceroleiras } \\
\cline { 2 - 8 } & TMFR & DMFR & VITC & BRIX & pH & RMP20FR & PMFR & TCOLH \\
\hline TMIN2 & $0,22 \mathrm{NS}$ & $0,30 \mathrm{NS}$ & $-0,31 \mathrm{NS}$ & $-0,52^{* *}$ & $-0,16 \mathrm{NS}$ & $0,38^{*}$ & $0,23 \mathrm{NS}$ & $-0,35 \mathrm{NS}$ \\
\hline PR & $0,34 \mathrm{NS}$ & $0,40^{*}$ & $0,002 \mathrm{NS}$ & $-018 \mathrm{NS}$ & $0,26 \mathrm{NS}$ & $0,50^{* *}$ & $0,35 \mathrm{NS}$ & $0,04 \mathrm{NS}$ \\
\hline HL & $0,15 \mathrm{NS}$ & $0,19 \mathrm{NS}$ & $0,09 \mathrm{NS}$ & $-0,03 \mathrm{NS}$ & $-0,13 \mathrm{NS}$ & $0,03 \mathrm{NS}$ & $0,19 \mathrm{NS}$ & $0,92^{* *}$ \\
\hline
\end{tabular}

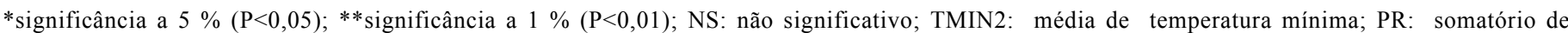

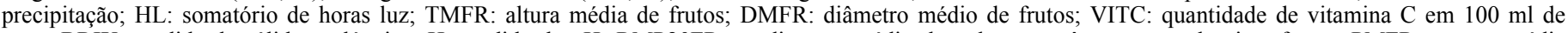

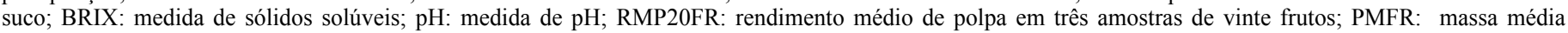
de frutos; TCOLH: tempo de colheita.

TABELA 2 - Correlações entre a média dos caracteres físico-químicos de frutos dentro de aceroleiras dos genótipos (CL2, CL5, CL11, R1 e R2) e as variáveis meteorológicas no período de dezembro/97 a janeiro/99, nas condições de Jaboticabal-SP.

\begin{tabular}{|c|c|c|c|c|c|c|c|c|c|c|c|c|c|c|c|}
\hline \multirow{3}{*}{$\begin{array}{c}\text { Caracteres } \\
\text { do } \\
\text { Fruto }\end{array}$} & \multicolumn{15}{|c|}{ Correlações dentro da média de genótipo } \\
\hline & \multicolumn{3}{|c|}{ Genótipo CL 2} & \multicolumn{3}{|c|}{ Genótipo CL 5} & \multicolumn{3}{|c|}{ Genótipo CL 11} & \multicolumn{3}{|c|}{ Genótipo R1 } & \multicolumn{3}{|c|}{ Genótipo R2 } \\
\hline & TMIN2 & PR & $\mathrm{HL}$ & TMIN2 & PR & $\mathrm{HL}$ & TMIN2 & PR & $\mathrm{HL}$ & TMIN2 & PR & $\mathrm{HL}$ & TMIN2 & PR & $\mathrm{HL}$ \\
\hline TMFR & $0,61 \mathrm{NS}$ & $0,22 \mathrm{NS}$ & $-0,46 \mathrm{NS}$ & $0,20 \mathrm{NS}$ & $0,08 \mathrm{NS}$ & $-0,08 \mathrm{NS}$ & $0,37 \mathrm{NS}$ & $0,76 \mathrm{NS}$ & $0,28 \mathrm{NS}$ & $0,67 \mathrm{NS}$ & $0,65 \mathrm{NS}$ & $0,24 \mathrm{NS}$ & $0,65 \mathrm{NS}$ & $0,39 \mathrm{NS}$ & $0,28 \mathrm{NS}$ \\
\hline DMFR & $0,55 \mathrm{NS}$ & $0,45 \mathrm{NS}$ & $-0,23 \mathrm{NS}$ & $0,58 \mathrm{NS}$ & $0,50 \mathrm{NS}$ & $0,58 \mathrm{NS}$ & $0,02 \mathrm{NS}$ & $0,63 \mathrm{NS}$ & $0,60 \mathrm{NS}$ & $0,62 \mathrm{NS}$ & $0,65 \mathrm{NS}$ & $0,32 \mathrm{NS}$ & $0,72 \mathrm{NS}$ & $0,44 \mathrm{NS}$ & $0,14 \mathrm{NS}$ \\
\hline VITC & $-0,61 \mathrm{NS}$ & $0,31 \mathrm{NS}$ & $0,59 \mathrm{NS}$ & $-0,58 \mathrm{NS}$ & $-0,53 \mathrm{NS}$ & $0,15 \mathrm{NS}$ & $-0,36 \mathrm{NS}$ & $0,04 \mathrm{NS}$ & $0,42 \mathrm{NS}$ & $-0,76 \mathrm{NS}$ & $-0,03 \mathrm{NS}$ & $-0,22 \mathrm{NS}$ & $-0,62 \mathrm{NS}$ & $0,23 \mathrm{NS}$ & $-0,09 \mathrm{NS}$ \\
\hline BRIX & $-0,54 \mathrm{NS}$ & $0,14 \mathrm{NS}$ & $0,75 \mathrm{NS}$ & $-0,77^{*}$ & $-0,25 \mathrm{NS}$ & $-0,07 \mathrm{NS}$ & $-0,79 \mathrm{NS}$ & $-0,29 \mathrm{NS}$ & $0,31 \mathrm{NS}$ & $-0,62 \mathrm{NS}$ & $0,17 \mathrm{NS}$ & $-0,13 \mathrm{NS}$ & $-0,55 \mathrm{NS}$ & $-0,17 \mathrm{NS}$ & $-0,51 \mathrm{NS}$ \\
\hline $\mathrm{PH}$ & $-0,14 \mathrm{NS}$ & $-0,001 \mathrm{NS}$ & $0,08 \mathrm{NS}$ & $-0,08 \mathrm{NS}$ & $0,51 \mathrm{NS}$ & $-0,48 \mathrm{NS}$ & $0,55 \mathrm{NS}$ & $0,95^{*}$ & $0,03 \mathrm{NS}$ & $-0,64 \mathrm{NS}$ & $0,01 \mathrm{NS}$ & $-0,31 \mathrm{NS}$ & $-0,24 \mathrm{NS}$ & $0,46 \mathrm{NS}$ & $-0,13 \mathrm{NS}$ \\
\hline RMP20FR & $0,63 \mathrm{NS}$ & $0,34 \mathrm{NS}$ & $-0,25 \mathrm{NS}$ & $0,71 \mathrm{NS}$ & $0,73 \mathrm{NS}$ & $-0,19 \mathrm{NS}$ & $0,29 \mathrm{NS}$ & $0,82^{*}$ & $0,34 \mathrm{NS}$ & $0,18 \mathrm{NS}$ & $0,63 \mathrm{NS}$ & $0,28 \mathrm{NS}$ & $0,40 \mathrm{NS}$ & $0,94^{* *}$ & $\overline{0,03 \mathrm{NS}}$ \\
\hline PMFR & $0,33 \mathrm{NS}$ & $0,24 \mathrm{NS}$ & $-0,23 \mathrm{NS}$ & $0,40 \mathrm{NS}$ & $0,41 \mathrm{NS}$ & $0,51 \mathrm{NS}$ & $0,24 \mathrm{NS}$ & $0,79 \mathrm{NS}$ & $0,40 \mathrm{NS}$ & $0,68 \mathrm{NS}$ & $0,62 \mathrm{NS}$ & $0,32 \mathrm{NS}$ & $0,59 \mathrm{NS}$ & $0,19 \mathrm{NS}$ & $0,41 \mathrm{NS}$ \\
\hline TCOLH & $-0,64 \mathrm{NS}$ & $0,12 \mathrm{NS}$ & $0,93^{* *}$ & $-0,31 \mathrm{NS}$ & $-0,44 \mathrm{NS}$ & $0,83^{*}$ & $-0,59 \mathrm{NS}$ & $0,04 \mathrm{NS}$ & $0,95^{* *}$ & $0,15 \mathrm{NS}$ & $-0,12 \mathrm{NS}$ & $0,93^{* *}$ & $-0,4 \perp N S$ & $-0,14 \mathrm{NS}$ & $0,90^{* *}$ \\
\hline
\end{tabular}

*significância a 5 \% ( $<<0,05)$; **significância a $1 \%(\mathrm{P}<0,01)$; NS: não significativo; TMIN2: média de temperatura mínima; PR: somatório de precipitação; HL: somatório de horas luz; TMFR: altura média de frutos; DMFR: diâmetro médio de frutos; VITC: quantidade de vitamina C em $100 \mathrm{ml}$ de suco; BRIX: medida de sólidos solúveis; pH: medida de pH; RMP20FR: rendimento médio de polpa em três amostras de vinte frutos; PMFR: massa média de frutos; TCOLH: tempo de colheita.

colheita, incluindo-se três dias antecedentes, apresentou também correlações positivas com altura, diâmetro, rendimento médio de polpa e massa média de frutos, merecendo atenção os coeficientes de 0,40 na correlação de LMFR com PR e de 0,50 na correlação de RMP20FR com PR, ambos significativos a 5 e $1 \%$ de probabilidade, respectivamente (Tabela 1). Nota-se ainda que a correlação entre RMP20FR com PR, dentro do genótipo CL11, foi de 0,82 e de 0,94 dentro do R2, ambas significativas (Tabela 2). Na média entre os genótipos, quanto aos caracteres tecnológicos de fruto, VITC e pH, verifica-se que as correlações com PR são positivas, enquanto a correlação de ${ }^{\circ}$ BRIX com PR é negativa (Tabela 1). Destaca-se ainda, dentro do CL11, a correlação de $\mathrm{pH}$ com PR de 0,95 , significativa.

As correlações do somatório de horas-luz diárias (HL) obtidas no período de colheita, incluindo-se os três dias antecedentes com a média dos genótipos nas variáveis TMFR, LMFR, VITC, RMP20FR e PMFR, foram positivas (Tabela 1), concordando com os resultados de vários autores (Murphy, 1939; Harding et al. , 1939; Winstron, 1947; Brown, 1955; e Nakasone et al., 1966), quando afirmaram que a produção de ácido ascórbico é bastante influenciada pela radiação solar e, com os dados de Aróstegui et al. (1955), que observaram relação entre os fatores climáticos com o conteúdo de vitamina $\mathrm{C}$ em frutos de acerolas colhidas em épocas distintas. Contudo, observa-se que, entre as variáveis físico-químicas de frutos de aceroleiras nas variáveis TMFR, LMFR, VITC, RMP20FR e PMFR da Tabela 2, a depender do genótipo, podem apresentar correlações com HL positivas ou negativas, sugerindo uma resposta diferenciada de genótipo a genótipo e possivelmente outros fatores. Isso, em parte, explica o fato de os genótipos CL2, CL5 e R2 frutificarem plenamente em maio/98, mês frio nas condições de Jaboticabal, com temperatura mínima diária oscilando entre 10 e $16,9^{\circ} \mathrm{C}$, enquanto os genótipos R1 e CL11 frutificaram em abril/98, estendendo a colheita aos primeiros cinco dias de maio. Nota-se, ainda, que os genótipos estudados não frutificaram de junho a agosto/98, quando a temperatura máxima diária do período variou entre 20 e $32,5^{\circ} \mathrm{C}$, a mínima entre 8,3 e $19^{\circ} \mathrm{C}$ e a média entre 13,5 e $24,5^{\circ} \mathrm{C}$, ratificando a escolha da média de temperatura mínima obtida no período da colheita, incluindose os três dias antecedentes (TMIN2) como a de maior influência nas variáveis físico-químicas dos frutos de aceroleiras, uma vez que é a temperatura mínima que condiciona o crescimento vegetativo da aceroleira e sua conseqüente produção de frutos, induzindo-a ao repouso ou à produção. Isso concorda com Teixeira et al. (1995), quando classificaram como ótima para o desenvolvimento e produtividade comercial da aceroleira a faixa de temperatura entre 25 e $27^{\circ} \mathrm{C}$, com uma temperatura média igual ou superior a $20^{\circ} \mathrm{C}$ e uma temperatura média do mês mais frio maior ou igual a $14^{\circ} \mathrm{C}$.

Quanto ao tempo de colheita em dias (TCOLH), observase, na Tabela 1 , o valor de $-0,35$ na correlação com TMIN2, refletindo influência dessa temperatura na extensão do período de colheita e que PR e HL, correlacionaram-se positivamente com TCOLH na média dos genótipos, destacando o coeficiente de correlação de 0,92 entre HL e TCOLH, significativo a $1 \%$ de probabilidade, demonstrando que a insolação, medida em horas diárias, aliada à precipitação do período, além de influenciar na produção de ácido ascórbico, conforme Murphy (1939), Harding et al. (1939), Winstron (1947) e Brown (1955), influencia também nos demais caracteres físico-químicos dos frutos e na extensão das safras (Jackson e Pennock, 1958).

Por fim, o somatório de horas-luz diárias (HL) obtida no período de colheita, juntamente com os três dias antecedentes, reflete, entre e dentro de todos os genótipos estudados, uma 
associação significativa com TCOLH, explicando em parte a periodicidade e a extensão das colheitas (safras) nos genótipos.

\section{CONCLUSÕES}

1) A média de temperatura mínima (TMIN2) e a precipitação (PR) associam-se positivamente com os caracteres físico-químicos dos frutos: altura média, diâmetro, rendimento médio de polpa e massa média; Vitamina $\mathrm{C} \mathrm{e} \mathrm{pH} \mathrm{correlacionam-se} \mathrm{negativamente}$ com TMIN2 e positivamente com PR. ${ }^{\circ}$ Brix correlaciona-se negativamente com TMIN2 e PR.

2) Ocorrem respostas diferenciadas dos genótipos a TMIN2, PR e HL.

3) Na média geral, as correlações das variáveis TMFR, LMFR, VITC, RMP20FR e PMFR com HL são positivas.

4) A média de temperatura mínima (TMIN2) é a temperatura mais influente nos caracteres físico-químicos dos frutos de aceroleiras. 5) As correlações de HL com TCOLH, entre e dentro de todos os genótipos, são direta e altamente significativas.

6) A insolação em horas-luz diárias (HL) condiciona a periodicidade e extensão das colheitas.

7) Ocorrem inter-relações entre as medidas meteorológicas, de modo que as influências são totalmente independentes.

\section{REFERÊNCIAS BIBLIOGRÁFICAS}

ARÓSTEGUI, F.; ASENJO, C.F.; MUÑIZ, A.I. \& ALEMAÑY, L. Observations and data on a promising selection of the West Indian Cherry, Malpighia punicifolia L. The Journal of Agriculture of the University of Puerto Rico. v.39, n.2, p.51-56, 1955.

ASENJO, C.F. Aspectos quimicos y nutritivos de la acerola (Malpighia punicifolia L.). Ciências, México, n.19, p.109-118, 1959.

BROWN, G.B. The ascorbic acid content of the tomatoes as related to ilumination. Proceedings of the American Society for Horticultural Science, Geneva, v.68, n.65, p.342-347, 1955.
GONZÁLEZ, A.M. Biologia floral e caracterização físicoquímica dos frutos de dois acessos de Passiflora cincinnata Mast. nas condições de Jaboticabal. Jabotical, SP. 1996. 80p. (Trabalho apresentado à Faculdade de Ciências Agrárias e Veterinárias, UNESP, Câmpus de Jaboticabal, para a obtenção do título de Mestre em Agronomia).

HARDING, P.L.; WINSTRON, J.R.; FISHER, D.F. Seasonal changes in the ascorbic acid content of juice of Florida oranges. Proceedings of the American Society for Horticultural Science, Geneva, v.36, n.3, p.358-370, 1939.

INSTITUTO ADOLFO LUTZ. Normas análiticas do Instituto Adolfo Lutz: métodos químicos e físicos para análise de alimentos. 2 ed. São Paulo, 1985. v.1, 371p.

JACKSON, G.C. \& PENNOCK, W. Fruit vitamin C production of five and six years old acerola trees. Journal of Agriculture of the University of Puerto Rico, Rio Piedras, v.42, p. 196-205, 1958. MURPHY, E. Vitamin C and light. Proceedings of the American Society for Horticultural Science, Geneva, v.36, n.3, p.498-499, 1939.

NAKASONE, H.Y.; MIYASHITA, R.K.; YAMANE, G.H. Factors affecting ascorbic acid content of the acerola (Malpighia glabra L.). Proceedings of the American Society for Horticultural Science, Geneva, v.89, p.161-166, 1966.

PEARSON, D. Laboratory Techniques in Food Analysis. London: London Butterwoths, 1973, p.58-60.

SAS/STAT. User Guide (Release 6.11). SAS Inst. Cary, NC., 1995. TEIXEIRA, A.H. DE C. \& AZEVEDO, P.V. de. Índice-limite do clima para o cultivo da acerola. Pesquisa Agropecuária Brasileira, Brasília, v.30, n.12, p.1403-1410, 1995.

WINSTRON, J.R. Vitamin C content and juice quality of exposed and shaded citrus fruits. Proceedings of the Florida State Horticulture Society, Florida, v.52, n.60, p.63-67, 1947. 